\title{
Enhancing the Visibility of Vernier Effect in a Tri-Microfiber Coupler Fiber Loop Interferometer for Ultrasensitive Refractive Index and Temperature sensing
}

\author{
Fangfang Wei \\ Photonics Research Centre, School of Electrical and Electronic Engineering, Technological University \\ Dublin, Kevin St, D08 NF82, Ireland, fangfang.wei@tudublin.ie
}

Dejun Liu

Shenzhen University, dejun.liu@szu.edu.cn

Zhe Wang

Technological University Du, Zhe.Wang@mytudublin.ie

See next page for additional authors

Follow this and additional works at: https://arrow.tudublin.ie/engscheleart2

Part of the Electromagnetics and Photonics Commons, Environmental Monitoring Commons, and the Optics Commons

\section{Recommended Citation}

F. Wei et al., "Enhancing the visibility of Vernier effect in a tri-microfiber coupler fiber loop interferometer for ultrasensitive refractive index and temperature sensing," Journal of Lightwave Technology, doi: 10.1109/JLT.2020.3035655.

This Article is brought to you for free and open access by the School of Electrical and Electronic Engineering at ARROW@TU Dublin. It has been accepted for inclusion in Articles by an authorized administrator of ARROW@TU

Dublin. For more information, please contact

arrow.admin@tudublin.ie, aisling.coyne@tudublin.ie, gerard.connolly@tudublin.ie.

Funder: TU Dublin

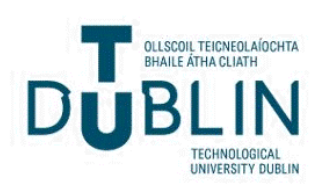




\section{Authors}

Fangfang Wei, Dejun Liu, Zhe Wang, Zhuochen Wang, Gerald Farrell, Qiang Wu, Gang-ding Peng, and Yuliya Semenova 


\title{
Enhancing the visibility of Vernier effect in a tri-microfiber coupler fiber loop interferometer for ultrasensitive refractive index and temperature sensing
}

\author{
Fangfang Wei, Dejun Liu, Zhe Wang, Zhuochen Wang, Gerald Farrell, Qiang Wu, Gang-Ding Peng, \\ Yuliya Semenova
}

\begin{abstract}
In this paper a Vernier effect based sensor is analyzed and demonstrated experimentally in a tri-microfiber coupler (Tri-MFC) and polarization-maintaining fiber (PMF) loop interferometer (Tri-MFC-PMF) to provide ultrasensitive refractive index and temperature sensing. The main novelty of this work is an analysis of parameters of the proposed Tri-MFC-PMF with the objective of determining the conditions leading to a "strong" Vernier effect. It has been identified by simulation that the Vernier effect is a primary factor in the design of Tri-MFC-PMF loop sensing structure for sensitivity enhancement. It is furthermore demonstrated experimentally that enhancing the visibility of the Vernier spectrum in the Tri-MFC-PMF allows to achieve an ultrahigh refractive index and temperature sensitivity with improved measurement accuracy. Specifically it is shown that small values of the total phase difference $(\pi / 16+N \pi) \sim(\pi / 4+N \pi)$, where $N$ is an integer, accumulated over the PMF loop and Tri-MFC loop result in a "strong" Vernier effect. Experimentally an ultrahigh refractive index sensitivity of $\mathbf{- 2 0 5 8 8} \mathbf{n m} / \mathrm{RIU}$ and temperature sensitivity of $0.019 \mathrm{~nm} /{ }^{\circ} \mathrm{C}$ are demonstrated by utilizing the stronger Vernier effect with "clear" Vernier spectrum. This analysis of the
\end{abstract}

Manuscript received $1^{\text {st }}$ September 2020; revised $29^{\text {th }}$ September 2020; accepted $30^{\text {th }}$ October 2020. Date of publication XXXX; date of current version XXXX. This work was supported in part by FIOSRAIGH 2016 (Dean of Graduate Students Award), TU Dublin, Ireland, in part by Guangdong Basic and Applied Basic Research Foundation (2019A1515110320), in part by Shenzhen Basic Research Foundation (JCYJ20190808173401660). (Corresponding author: Fangfang Wei.)

F. Wei, Z. Wang, Z. C. Wang, G. Farrell, and Y. Semenova are with the Photonics Research Centre, School of Electrical and Electronic Engineering, Technological University Dublin, Kevin St., Dublin 8, Ireland (e-mail: fangfang.wei@tudublin.ie;

D18130040@mytudublin.ie

yuliya.semenova@tudublin.ie).

D. Liu is with College of Optoelectronic Engineering, Shenzhen University, Shenzhen, Guangdong 518060, China (e-mail: dejun.liu@ szu.edu.cn).

Q. Wu is with the Department of Mathematics, Physics and Electrical Engineering, Northumbria University, Newcastle Upon Tyne, NE1 8ST, United Kingdom, and also with the Key Laboratory of Nondestructive Test (Ministry of Education) of Nanchang Hangkong University, Nanchang 330063, China (e-mail: qiang.wu@ northumbria.ac.uk).

G. D. Peng. is with the Photonics \& Optical Communications, School of Electrical Engineering \& Telecommunications, University of New South Wales, Sydney 2052, NSW, Australia (e-mail: g.peng@unsw.edu.au).

Color versions of one or more of figures in this paper are available online at http://ieeexplore.ieee.org.

Digital Object Identifier 10.1109/JLT.2020.XXXX. parameters may be useful to future researchers seeking to increase the measurement accuracy of sensors by enhancing the spectral visibility of the Vernier effect in other types of fiber optic interferometers.

Index Terms-tri-microfiber coupler, fiber loop interferometer, refractive index, temperature, Vernier effect

\section{INTRODUCTION}

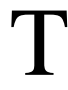
HE Vernier effect has attracted significant research interest in optical fiber sensing recently and has been used to increase the measurement sensitivity of sensors by taking advantage of the small-scale difference between two optical fiber interferometers [1][2][3][4][5].

The Vernier effect, originally employed in a mechanical Vernier caliper to enhance the accuracy of length measurement, has also been recently used to increase sensitivity in optical fiber sensing by taking advantage of the small-scale difference between two interferometers, which are used as the main ruler and the Vernier, respectively [1]. Several structures have been proposed to realize the Vernier effect, such as two cascaded Mach-Zehnder interferometers [2], optical microfiber coupler modal interferometers [3], two cascaded Fabry-Perot Interferometers [4], reflective $\theta$-shape microfiber resonator [5] and multimode Fabry-Perot Interferometer [6][7]. It should be noted that in all of the above structures in order to utilize the Vernier effect, the optical lengths of the reference arm and the sensing arm were carefully controlled.

In optical fiber sensing, the basic component of a Vernier effect scheme is an interferometric optical fiber structure. By carefully selecting an appropriate cascade of structures as well as their parameters, a 'strong' Vernier effect can be achieved leading to a large increase in the sensor sensitivity [7][8], an improvement which can potentially be applied to a wide range of applications currently using the optical fiber interferometers, including environmental monitoring, Earth geology, biological detection, aerospace and navigation [9][10][11][12][13]. Optical fiber interferometers based on Sagnac loops are especially attractive due to their simple fabrication and due to the fact that other devices can be easily incorporated into the loop in order to modulate the output spectrum. A classic Sagnac 
loop is formed by an optical fiber coupler which splits the input light into two beams which then counter-propagate within the polarization-maintaining fiber (PMF) loop and accumulate a phase difference before re-combining at the coupler and interfering with each other due to the acquired phase difference [14][15]. The Vernier effect was achieved by angle shift splicing two PMFs in a single Sagnac interferometer loop and the temperature sensitivity was $-2.44 \mathrm{~nm} /{ }^{\circ} \mathrm{C}$, which is 14.96 times greater compared with the Sagnac sensor without the Vernier effect $\left(-0.163 \mathrm{~nm} /{ }^{\circ} \mathrm{C}\right)$ [16].

Recent progress in microfiber fabrication opened the possibility to replace the traditional optical fiber couplers in the Sagnac loop with microfiber couplers (MFCs) in order to design highly sensitive sensors that take advantage of the evanescent field of the MFC for monitoring of ambient refractive index (RI) changes [17][18][19]. In the coupling region, continuous power distribution changes along the coupler cross section, which results in a different power splitting at the output ports while the coupling length and the refractive index are dependent both on the refractive index and temperature of the surrounding medium. Thermometers based on microfiber couplers have been shown to be able to measure temperature in excess of $1000{ }^{\circ} \mathrm{C}$ [20]. Fiber Bragg gratings (FBGs) are also common too be used for temperature sensing [21]. The use of a PMF loop in combination with an MFC based sensor offers the advantage of tunability of the free spectral range (FSR) of the transmission spectrum by adjusting the length of the PMF section. Ambient RI changes in such a configuration impact both of the beams counter-propagating through the MFC and influence the output interference spectrum of the PMF loop. For example, $\mathrm{Zu}$ et al. proposed an MFC in a Sagnac loop for detection of water pollutants with sensitivity of $423 \mathrm{pm} / \%$ [ [9]. Bai et al. proposed a microfiber Sagnac loop for simultaneous measurement of temperature and relative humidity, and demonstrated the sensitivities of 176.6 $\mathrm{pm} / \% \mathrm{RH}$ and $-123.5 \mathrm{pm} /{ }^{\circ} \mathrm{C}$ respectively [22]. Chen et al. demonstrated a highly sensitive micro-force sensor based on an MFC with sensitivity of $\sim 3754 \mathrm{~nm} / \mathrm{N}$. In our previous work [23], we reported a Tri-MFC based Sagnac loop for sensing of magnetic field, where changes of the RI of the magnetic fluid surrounding the MFC were used to determine the magnetic field intensity.

In this work, the structure is similar to our previous Tri-MFC-PMF loop structure [23] but the main novelty of this work is determining the condition of total phase delay accumulated over the PMF loop and Tri-MFC loop, to achieve a "strong" Vernier effect and furthermore providing an experimental demonstration that a high RI sensitivity with "clear" Vernier effect for the sensor can be achieved by optimizing the total phase delay of the Tri-MFC-PMF sensor. It has been established that the "strength" of Vernier effect can be directly related to and regulated by the total accumulated phase difference in the structure. To the best of our knowledge this is the first time such an analysis has been carried out and it may be useful to future researchers seeking to increase measurement accuracy by enhancing spectral visibility of the Vernier effect in other types of fiber optic interferometers.

\section{SIMULATION AND ANALYSIS OF THE SENSOR PARAMETERS}

Figure 1 shows a schematic diagram of the sensor. Since the structure is similar to that previously reported by us in [23] it will not be expanded upon further here. The waist section of tri-MFC was straight and suspended above the glass surface using two spacers fixed at both ends of a glass slide. This method ensures the mechanical stability of the sensor while maintaining physical contact with the environment whose RI is being measured.

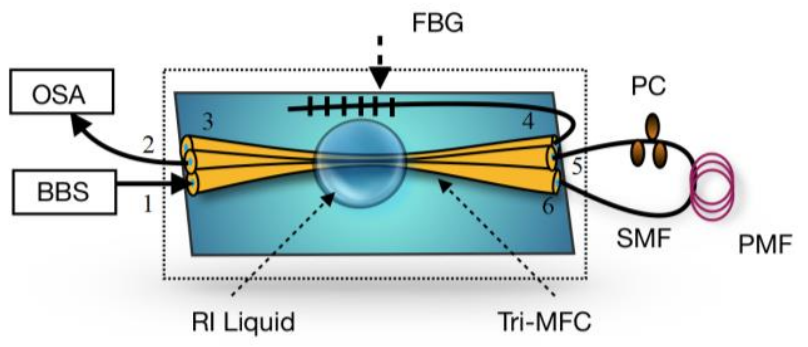

Fig. 1. Schematic diagram of the sensing scheme.

Based on the analytical expression derived in our previous work [23], under particular RI and temperature conditions, the expanded transmission throughput from port 1 to port 2 via the Tri-MFC-PMF loop can be described by substituting the coupling ratio equation into the classic PMF loop equation as:

$T=\sin ^{2}\left(k l_{e f f}\right)\left(1-2 \sin ^{2}\left(k l_{e f f}\right)\right) \sin ^{2}(2 \theta) \cos ^{2}(\Delta \varphi)$

Where $k$ is the coupling coefficient of the MFC, $\Delta \varphi=$ $\left(\Delta \varphi_{P M F}+\Delta \varphi_{M F C}\right)$ is the total phase difference, and $\Delta \varphi_{P M F}=$ $2 \pi L\left(n_{0}-n_{e}\right) / \lambda$ is the phase difference accumulated within the PMF loop, $\Delta \varphi_{M F C}$ is the phase difference accumulated in the Tri-MFC, $\theta$ is the initial angle between light polarizations at each end of the PMF, $L$ is the length of the PMF, $\left(n_{o}-n_{e}\right)$ is the birefringence of the PMF and $\lambda$ is the operating wavelength. The coupling ratio $r$ can be calculated based on the value of coupling coefficient $k$, whose value varies along the length of the tapered region of the fiber coupler.

$$
r=\sin ^{2}\left(k l_{e f f}\right)
$$

As it was shown in [24], $k$ is also a function of the surrounding RI. $l_{\text {eff }}$ represents the effective interaction length of the Tri-MFC.

Considering the rotational symmetry and orthogonality between the normal modes in the Tri-MFC, we limit the study to the case where the three coupling coefficients $(k)$ are identical with the same value of $k$, with a phase difference of $\frac{\pi}{3}$ for the electrical field coupling from one microfiber to the other microfibers, that is $\varphi_{M F C}=\frac{\pi}{3}$. With the change of surrounding RI, the effective refractive indices of all microfibers will change. The phase difference $\Delta \varphi_{P M F}$ accumulated within the PMF loop is decided by the PMF length and the polarization 
state.

From equation (1), one can see that the resulting transmission spectrum and the 'strength' of the Vernier effect are due to the 'beating' of the different interferences within the different elements of the structure (represented by different sinusoidal functions), namely, within the Tri-MFC and PMF loop, for the two polarizations.

In Fig.1, the signals from port 5 and port 6 acquire a phase difference propagating through the PMF loop. After entering the Tri-MFC, the phase difference accumulated within its waist region can be calculated by:

$$
\Delta \varphi_{M F C}=\frac{\pi l_{e f f}^{5} \Delta n_{e f f}^{5}}{\lambda}-\frac{\pi l_{e f f}^{6} \Delta n_{e f f}^{6}}{\lambda}
$$

where $\Delta n_{e f f}^{5}, \Delta n_{e f f}^{6}$ are the effective refractive indices of the taper waist region connected to ports 5 and 6 respectively.

In order to determine the combination of the sensor structure parameters leading to an enhanced visibility of Vernier spectrum, we analyzed equation (1) using Matlab envelope function ([up lo] =envelope ( $\mathrm{x}$, 'peak'), which returns the upper ('up') and lower ('lo') peak envelopes of $\mathrm{x}$ ). In the analysis the MFC waist region length was fixed at $2 \mathrm{~mm}$, the waist diameter at $6 \mu \mathrm{m}$ (each microfiber radius was assumed as $1 \mu \mathrm{m}$ ), and the length of the PMF loop was set as $0.1 \mathrm{~m}$. To visualize the relationship between the total phase difference, FSR and the visibility of Vernier spectrum, the transmission spectra for different total phase differences of $\frac{\pi}{16}+N \pi, \frac{\pi}{2}+N \pi$ and $N \pi$, where $\mathrm{N}$ is an integer, were simulated with all other parameters fixed. The results of the simulations are shown in Fig. 2. In accordance with the schematic diagram of the structure shown in Fig. 1, port 5 and port 6 are the coupled port and throughput port of the MFC, respectively.

Fig. 2 (a, b and c) are the simulated spectra for the throughput port (black line) and coupled port (red line) of the MFC for the different values of total phase difference, $\Delta \varphi$, specifically $\frac{\pi}{16}+N \pi, \frac{\pi}{2}+N \pi$ and $N \pi$. Fig. 2 (a', b' and c') illustrate the corresponding Vernier spectra (at port 2 in Fig. 1) for these values of the total phase difference. When the total phase difference is small $\left(\frac{\pi}{16}+N \pi\right)$, a strong Vernier effect is observed. When the total phase difference increases to $\frac{\pi}{2}+N \pi$, the Vernier effect becomes weaker, the transmission spectrum exhibits isolated dips, in which the neighboring dips are inhibited. When the total phase difference is at a maximum $(N \pi)$, no Vernier effect is observed. Fig. 2 (d \& e) show the simulated spectra (at port 5) and Vernier spectra (at port 2) for two RI values with a 0.002 difference.

From Fig. 2 (a'- c'), one can see the double-periodicity within the transmission spectrum of the proposed sensor determined by the interaction of the interference within the PMF loop and within the Tri-MFC. The low frequency envelope curve, which we will henceforth refer to as the Vernier spectrum, is due to 'beating' of the different interferences, from the Tri-MFC and PMF loop, for the two polarizations, respectively. From the above analysis we can conclude that in our previous work [23] the Vernier effect was not observed because the combination of the various physical parameters of the sensing system results in a total phase difference accumulated within the PMF loop and the MFC which is close to $N \pi$, where $N$ is an integer. The significant distinction in this current work, is that the combination of the system parameters is deliberately chosen to provide a small total phase difference $\left(N \pi<\Delta \varphi<\frac{\pi}{2}+N \pi\right)$, so in turn the visibility of the Vernier effect is enhanced.

From Fig. 2 ( $\mathrm{d} \& \mathrm{e})$, the drifts of the two envelopes are different when the surrounding RI changes; this is due to the wavelength dependence of the FSR of the MFC spectrum. As was shown in ref [24], the FSR of an MFC increases toward shorter wavelengths.
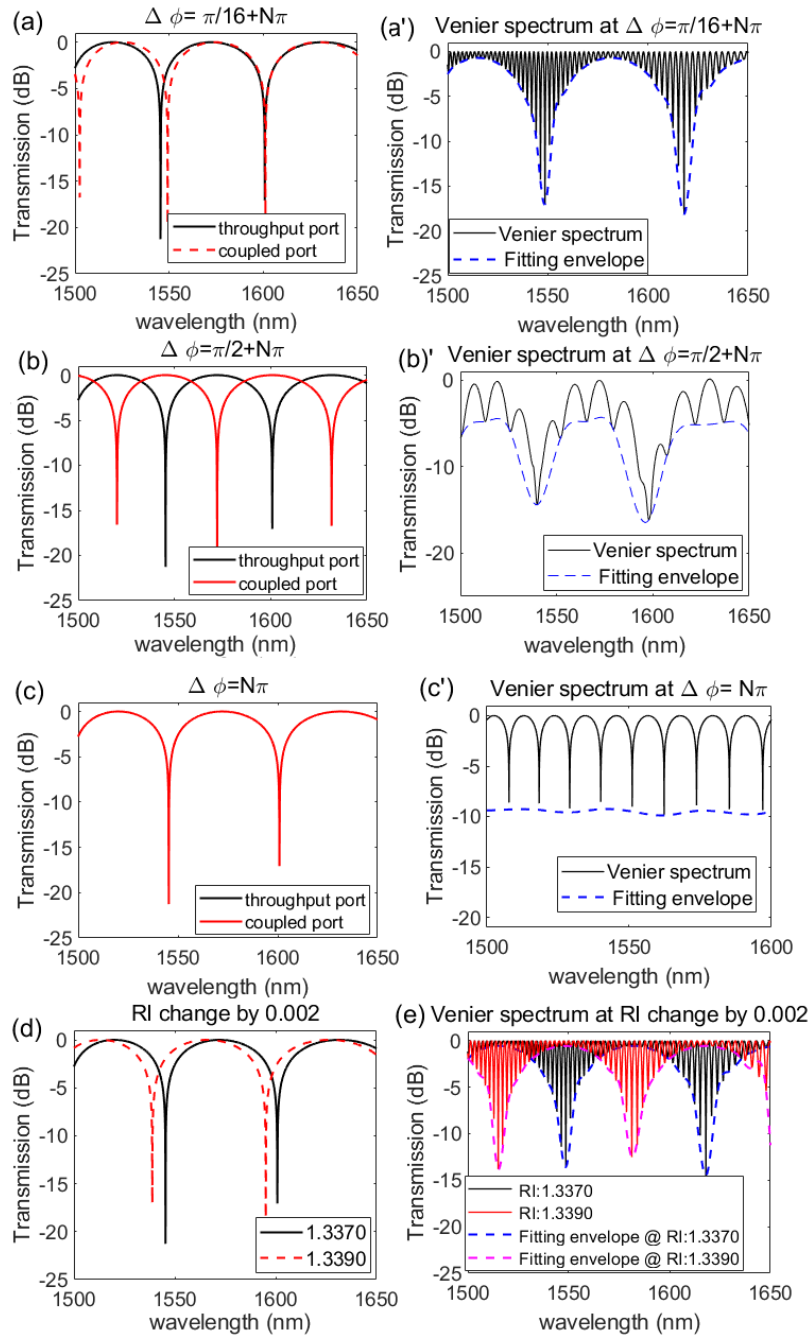

(e) Venier spectrum at RI change by 0.002

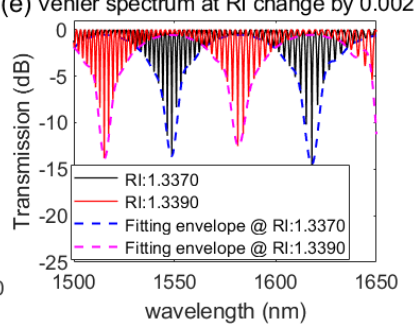

Fig. 2. (a-c) Simulated spectra at ports 5 (black line) and 6 (red line) for the different values of total phase difference $\left(\frac{\pi}{16}+N \pi, \frac{\pi}{2}+N \pi\right.$ and $N \pi$ ), where $N$ is an integer; (a'c') simulated Vernier spectra (at port 2) corresponding to total phase differences of $\frac{\pi}{16}+$ $N \pi, \frac{\pi}{2}+N \pi$ and $\mathrm{N}$, (d \& e) simulated spectra (at port 5) and Vernier spectra (at port 2) for two RI values with a 0.002 difference.

The proposed structure has the advantage of design flexibility so that an even higher RI sensitivity can be achieved by maximizing the visibility of Vernier spectrum. The total 
phase difference ( $\Delta \varphi$ in Eq. 1) comprises of the phase difference accumulated within the $\mathrm{PMF}$ loop $\left(\Delta \varphi_{\mathrm{PMF}}\right)$ and the phase difference within the MFC $\left(\Delta \varphi_{\mathrm{MFC}}\right)$. Thus, PMF and MFC equally contribute the total phase difference. In practice once the length and the type of the PMF are fixed, $\Delta \varphi_{\mathrm{PMF}}$ will be fixed. Since the light transmitted within PMF has no contact with surrounding material, the RI of surrounding material has no influence on the phase difference $\Delta \varphi_{\mathrm{PMF}}$. The phase difference $\Delta \varphi_{\mathrm{MFC}}$ is determined by both the geometrical parameters of the MFC and the refractive index of the surrounding material. Therefore only the $\Delta \varphi_{\mathrm{MFC}}$ component of the total phase difference is RI sensitive. Thus, replacement of the MFC with a traditional optical fiber coupler would result in the complete loss of sensitivity of the structure to the surrounding RI.

It should also be noted that since the total phase difference is the key parameter influencing the strength of Vernier effect in the proposed sensing structure, its optimal value leading to "strong" Vernier effect can be effectively modulated by PMF and MFC.

\section{EXPERIMENTAL RESULTS AND DISCUSSION}

The experimental setup has been described previously but in summary the Tri-MFC and the FBG are fixed on a glass slide to form the main sensing element of the sensor. The Tri-MFC is fabricated from three single mode fibers (SMF) fused and tapered together using the microheater brushing technique [25]. Two of the tree output ends of the Tri-MFC (ports 5 and 6) are connected with a section $(10 \mathrm{~cm})$ of PMF with a polarization controller (PC) included in the loop. The third output of the MFC (port 4) is connected to the FBG. The Tri-MFC is immersed in a liquid whose RI is being monitored.

The influence of the phase difference on the strength of Vernier effect is illustrated in Fig. 3 (a), the top part of which shows three types of experimental transmission spectra plotted for different values of the phase difference $\frac{\pi}{4}+N \pi, \frac{\pi}{2}+N \pi$ and $(N+1) \pi$. As one can see from the plot, the spectrum corresponding to the smallest phase difference (on the left) has a single most pronounced dip ('strong' Vernier effect), while the spectrum plotted for the largest phase difference (on the right) contains almost equal extinction ratio dips (weak Vernier effect). The smaller phase difference results a denser Vernier resonant spectrum, leading to a more accurate envelope fitting; while the larger phase difference results in a less dense Vernier spectrum, and thus less accurate envelope fitting. The bottom part of Fig. 3 (a) shows a normalized Vernier effect as a function of the phase difference, where ' 1 ' represents the strongest effect and ' 0 ' - the weakest effect. The orange triangle is the condition when the Vernier effect is strong, and the orange star is the condition in ref. [23] when no Vernier effect is observed.
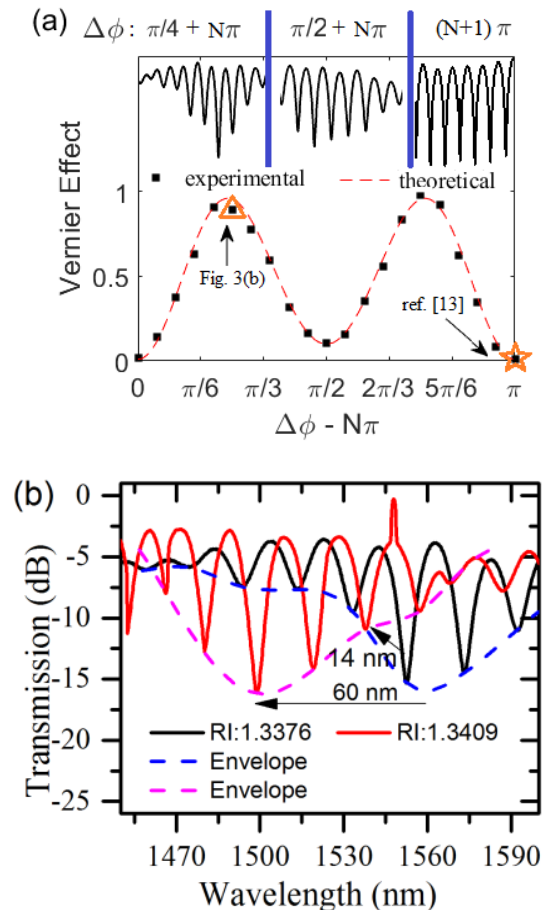

Fig. 3. (a) The normalized Vernier effect in experimental spectrum vs. phase difference $\Delta \varphi$ in the range of $(0, \pi)$ (the orange triangle is the condition when the Vernier effect is strong, and the orange star is the condition when no Vernier effect is observed as in ref. [23]); (b) experimental transmission spectra for two different ambient RIs.

The Vernier spectrum with respect to the total phase difference $\Delta \varphi$ has a modulated profile. The Vernier effect of each profile/ spectrum is characterized by an extinction ratio / contrast which can be defined as

$$
V E=\frac{\left(\text { High }_{\text {peak-to-peak }}\right)-\left(\text { Low }_{\text {peak-to-peak }}\right)}{\text { High }}
$$

The normalized Vernier Effect (NVE) is defined as

$$
N V E=\frac{\operatorname{VE}(\Delta \varphi)}{V E_{M a x}}=\frac{\operatorname{VE}(\Delta \varphi)}{V E\left(N \pi+\frac{\pi}{4}\right)}
$$

Fig. 3 (b) shows experimental transmission spectra for the MFC sample (diameter $6 \mu \mathrm{m}$, PMF length $0.1 \mathrm{~m}$ ) for two different values of the ambient RI. The corresponding Vernier envelopes (dashed lines) are plotted by fitting the minima of the constitute lower peaks, shown by the solid lines.

From Fig. 3 (b), the reference dip of the Tri-MFC blue-shifts by $14 \mathrm{~nm}$ as the RI increases by 0.0033 , while the envelope spectrum blue-shifts by $60 \mathrm{~nm}$, which is more than 4 times the Tri-MFC dip's shift, demonstrating the benefit of utilizing the Vernier effect The experimental spectra observed with the OSA are in a good agreement with the simulations in Fig. 2 (e).

It should be noted that the Tri-MFC transmission is wavelength dependent and the spectral distances between each pair of neighboring dips are slightly different. The FSR for the envelope depends on the beat frequency of the two superimposed waves determined by the interference within the 
Tri-MFC and the PMF loop.

It should be noted that the FBG peak is not visible in the case of the transmission spectrum for $\mathrm{RI}=1.3376$ (black line in Fig. 3 (b)) due to its closeness to a dip in the interference spectrum. This phenomenon is clearly illustrated in Fig. 4 (b). In practice this problem could be avoided by adjusting the polarization state of the incoming light with the help of polarization controller which should lead to a change in the relative position of the interference spectrum with respect to the FBG peak.
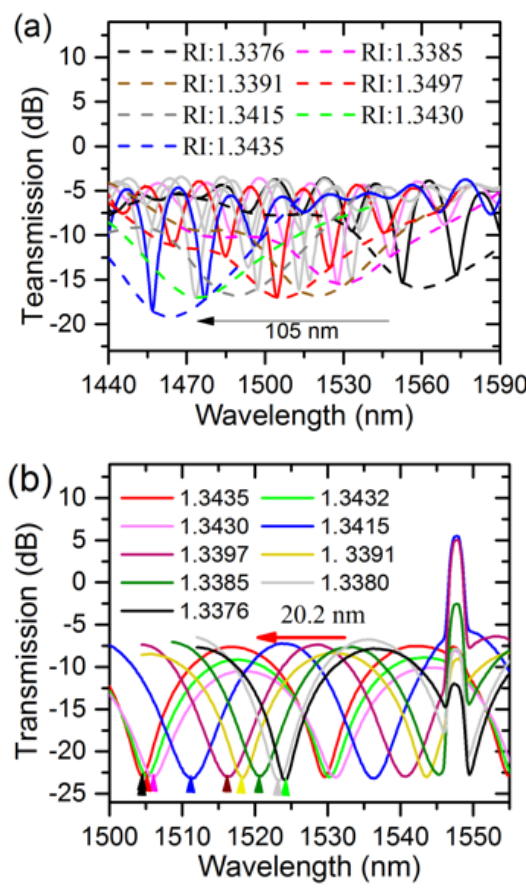

Fig. 4. (a) Experimental transmission spectra with RI changes shown in a wide spectral range; (b) the same experimental spectra for the envelope in region around $1550 \mathrm{~nm}$.

Fig. 4 (a) shows experimental Vernier spectra of the proposed sensor immersed in various calibrated RI liquids from 1.3376 to 1.3435. Fig. 4 (b) shows the same spectra in a narrow spectral interval within a single envelope in the vicinity of the spectral peak associated with the FBG.

As expected, in Fig. 4 (b) the spectral peak corresponding to the FBG remains constant regardless the variations in the surrounding RI, while the interference dips of the Tri-MFC (with a waist diameter of $6 \mu \mathrm{m}$ ) monotonically blue-shift by $20.2 \mathrm{~nm}$ when the RI value increases from 1.3376 to 1.3435 . The corresponding Vernier (envelope) spectrum is $\sim 100 \mathrm{~nm}$ wide.

Fig. 5 presents linear fittings of the spectral shifts for both the Tri-MFC and Vernier dips versus the liquid RI. From Fig.5, the sensitivities are estimated as $-4457.6 \mathrm{~nm} / \mathrm{RIU}$ for the Tri-MFC spectral shift and -20588 nm/RIU for the Vernier spectral shift, over an RI range from 1.3376 to 1.3435 . Refractive Index Unit (RUI) is the minimum detectable change in the refractive index. The term 'nm/RUI' represents the measurement unit for the total spectral shift with the change of refractive index.

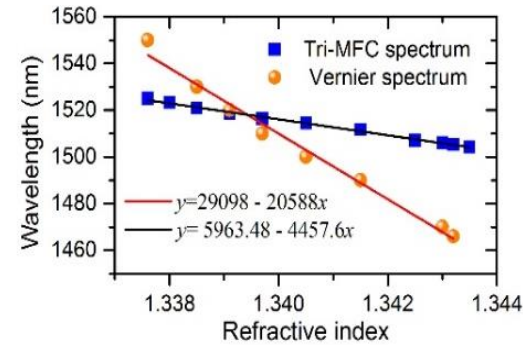

Fig. 5. Fitting of the spectral shift data for the dip at $1524.4 \mathrm{~nm}$ (red line) and the dip in the Vernier spectrum at $1550 \mathrm{~nm}$ (black line) when the RI changes from 1.3376 to 1.3435 .

It should be noted that although tracking of the Vernier dip involves an additional step of fitting the experimental spectrum with an envelope function, utilizing the Vernier effect is worthwhile as it allows for a significant improvement in the RI sensitivity of the proposed sensing structure.

The strong spectral peak in the experimental spectra at circa $1540 \mathrm{~nm}$ is associated with the FBG and its position is temperature dependent. The spectrum of the Tri-MFC-PMF loop is also influenced by temperature changes as illustrated in Fig. 6 (a). The Vernier spectrum is therefore also temperature dependent although this dependency is due to the temperature sensitivity of the Tri-MFC spectrum only since in the experiment the PMF loop was not subjected to temperature variations.

From Fig. 6 (a \& b) one can see that the FBG peak red-shifts by about $4.8 \mathrm{~nm}$ due to a temperature change from $20{ }^{\circ} \mathrm{C}$ to 260 ${ }^{\circ} \mathrm{C}$, the Tri-MFC dip drifts by $\sim 8.8 \mathrm{~nm}$ towards shorter wavelengths while the Vernier dip also shifts towards shorter wavelengths by about $45 \mathrm{~nm}$.
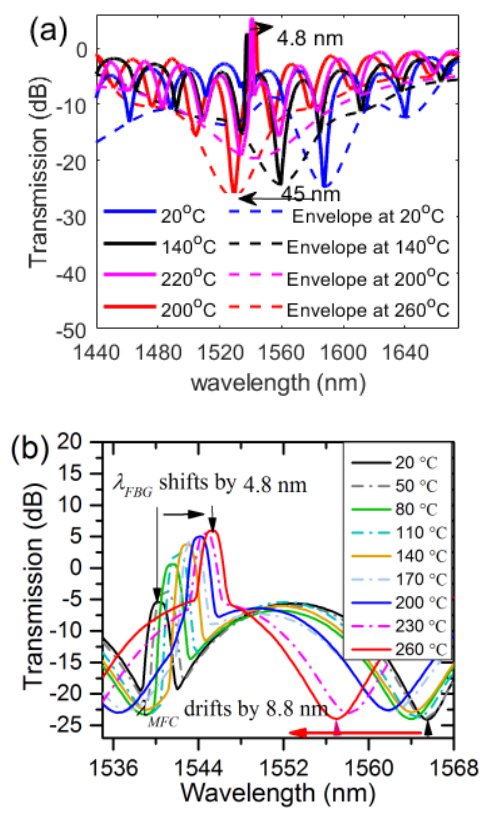

Fig. 6. (a) Experimental Vernier transmission spectrum (envelope) when the surrounding environment temperature increases; (b) spectral responses of the FBG peak and Tri-MFC-PMF corresponding to different temperatures ranging from $20^{\circ} \mathrm{C}$ to $260^{\circ} \mathrm{C}$.

Fig. 7 shows the experimental temperature responses, with curve fitting for the FBG, Tri-MFC and Vernier spectra versus 
temperature in the range from $20{ }^{\circ} \mathrm{C}$ to $260{ }^{\circ} \mathrm{C}$. As one can see from the graph, all of the above spectral features are temperature dependent with the FBG peak and Vernier dip displaying linear dependencies and the Tri-MFC dip showing slightly non-linear behaviour.

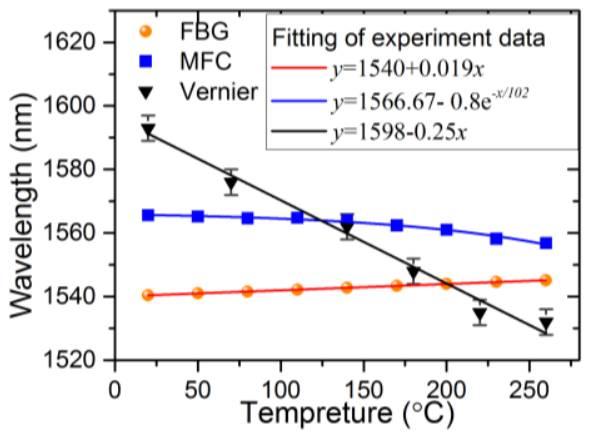

Fig. 7. Temperature responses of the FBG, Tri-MFC and Vernier spectra.

From Fig.7, the MFC-related spectral shift becomes nonlinear at higher temperatures (above $150{ }^{\circ} \mathrm{C}$ ). This is due to the difference in the thermal expansion coefficient of the silica and the thermo-optic coefficient in the tapered fiber [26] over the range of temperatures from 20 to $260{ }^{\circ} \mathrm{C}$.

As has been demonstrated in several published studies, the proposed sensing structure offers the advantage of a very high refractive index sensitivity combined with temperature self-referencing capability. both the surrounding RI and temperature can be determined from the output spectrum of the proposed sensor using the characteristic matrix approach with the coefficients derived from the above experiments thus:

$$
\left[\begin{array}{c}
\Delta \lambda_{F B G} \\
\Delta \lambda_{\text {Vernier }}
\end{array}\right]=\left[\begin{array}{cc}
0 & 0.019 \\
-20588 & -0.25
\end{array}\right] \cdot\left[\begin{array}{c}
\Delta R I \\
\Delta T
\end{array}\right]
$$

where $\Delta \lambda_{F B G}$ and $\Delta \lambda_{V e r n i e r}$ are the spectral shifts (measured in $\mathrm{nm}$ ) associated with the FBG and Vernier transmission spectrum, and $\Delta \mathrm{RI}$ and $\Delta \mathrm{T}$ are the corresponding changes in the $\mathrm{RI}$ and temperature, which caused the spectral shifts. It should be noted that while it is possible to use the data for the wavelength shift of the Tri-MFC dip $\left(\Delta \lambda_{M F C}\right)$ in the characteristic matrix, that would lead to a lower RI sensitivity. In addition, due to the non-linear nature of the temperature dependence of the Tri-MFC spectrum the characteristic matrix would become nonlinear.

We carried out the experiment several times for increasing/decreasing RI and temperature values and both the fringe shifts and the Vernier spectrum envelope shifts showed excellent repeatability, similar to that demonstrated in our previous work [23].

\section{CONCLUSION}

In summary, ultra-high sensitivity $\mathrm{RI}$ and temperature sensing utilizing the Vernier effect is demonstrated experimentally, based on a Tri-MFC and PMF loop. The sensor was fabricated by connecting two ends of a Tri-MFC with PMF to form a loop. An FBG was connected to the extra end to provide RI-independent temperature information. An analysis of the parameters of the proposed sensing structure was carried out to determine the conditions leading a "strong" Vernier effect. It has been experimentally demonstrated that the Vernier effect can be critical to the sensing performance of the structure and its "strength" can be effectively modulated by PMF and MFC by comparison with the case when a 'weak' Vernier effect [23] is present. It has been found that small values of the total phase delay $(\boldsymbol{\pi} / \mathbf{1 6}+\boldsymbol{N} \boldsymbol{\pi}) \sim(\boldsymbol{\pi} / \mathbf{4}+\boldsymbol{N} \boldsymbol{\pi})$, where $\mathrm{N}$ is an integer, accumulated over the PMF loop and Tri-MFC loop, accumulated by the propagating light over the proposed sensor structure, resulted in 'strong' Vernier effect. The Vernier spectrum envelope of the sensor blue-shifts almost 5 times faster compared to that of the high frequency spectrum associated with the Tri-MFC. A maximum RI sensitivity of $-20588 \mathrm{~nm} / \mathrm{RIU}$ in the RI range from 1.3376 to 1.3435 was achieved along with temperature self-referencing capability. The proposed sensing structure also simultaneously provided temperature information with a sensitivity of $0.019 \mathrm{~nm} /{ }^{\circ} \mathrm{C}$ for FBG and $-0.036 \mathrm{~nm} /{ }^{\circ} \mathrm{C}$ for the $\mathrm{MFC}$ in the range from $20^{\circ} \mathrm{C}$ to $260{ }^{\circ} \mathrm{C}$, which can be further and significantly reduced with the capability of temperature self-referencing. The high demonstrated sensitivity to RI and the capability of simultaneously providing temperature information make the proposed sensor potentially attractive for applications in biosensing.

\section{REFERENCES}

[1] T. Claes, W. Bogaerts, and P. Bienstman, 'Experimental characterization of a silicon photonic biosensor consisting of two cascaded ring resonators based on the Vernier-effect and introduction of a curve fitting method for an improved detection limit', Opt. Express, 2010, doi: 10.1364/oe.18.022747.

[2] X. Jiang, Y. Chen, F. Yu, L. Tang, M. Li, and J.-J. He, 'High-sensitivity optical biosensor based on cascaded MachZehnder interferometer and ring resonator using Vernier effect', Opt. Lett., vol. 39, no. 22, p. 6363, 2014, doi: 10.1364/ol.39.006363.

[3] K. Li et al., 'Birefringence induced Vernier effect in optical fiber modal interferometers for enhanced sensing', Sensors Actuators, B Chem., 2018, doi: 10.1016/j.snb.2018.08.027.

[4] J. Tian, Z. Li, Y. Sun, and Y. Yao, 'High-Sensitivity Fiber-Optic Strain Sensor Based on the Vernier Effect and Separated Fabry-Perot Interferometers', J. Light. Technol., vol. 37, no. 21, pp. 5609-5618, 2019, doi: 10.1109/JLT.2019.2936174.

[5] Z. Xu, Y. Luo, D. Liu, P. P. Shum, and Q. Sun, 'Sensitivity-controllable refractive index sensor based on reflective $\theta$-shaped microfiber resonator cooperated with Vernier effect', Sci. Rep., vol. 7, no. 1, pp. 1-8, 2017, doi: 10.1038/s41598-017-10163-x.

[6] A. D. Gomes et al., 'Multimode fabry-perot interferometer probe based on vernier effect for enhanced temperature sensing', Sensors (Switzerland), 2019, doi: 10.3390/s19030453.

[7] L. Y. Shao et al., 'Sensitivity-enhanced temperature sensor with cascaded fiber optic Sagnac interferometers based on Vernier-effect', Opt. Commun., 2015, doi: 10.1016/j.optcom.2014.09.075.

[8] B. Wu, L. Chen, C. Zhao, and C. C. Chan, 'Polypyrrole-Coated Polarization Maintaining Fiber-Based Vernier Effect for Isopropanol Measurement', J. Light. Technol., 2019, doi: 10.1109/JLT.2019.2906648.

[9] L. Zu, H. Zhang, Y. Miao, B. Li, and J. Yao, 'Microfiber coupler with a Sagnac loop for water pollution detection', Appl. Opt., 2019, doi: 10.1364/ao.58.005859.

[10] X. Li, S. C. Warren-Smith, H. Ebendorff-Heidepriem, Y. N. Zhang, and L. V. Nguyen, 'Optical Fiber Refractive Index Sensor with Low Detection Limit and Large Dynamic Range Using a Hybrid Fiber Interferometer', J. Light. Technol., 2019, doi: 10.1109/JLT.2019.2908023. 
F. Wei et al., 'Magnetic field sensor based on a combination of a microfiber coupler covered with magnetic fluid and a Sagnac loop', Sci. Rep., 2017, doi: 10.1038/s41598-017-05199-y.

[12] M. Rajibul Islam, M. Mahmood Ali, M. H. Lai, K. S. Lim, and H. Ahmad, 'Chronology of fabry-perot interferometer fiber-optic sensors and their applications: A review', Sensors (Switzerland). 2014, doi: 10.3390/s140407451.

[13] N. Alberto, M. F. Domingues, C. Marques, P. André, and P. Antunes, 'Optical fiber magnetic field sensors based on magnetic fluid: A review', Sensors (Switzerland), vol. 18, no. 12, 2018, doi: $10.3390 / \mathrm{s} 18124325$

[14] L. P. Sun, J. Li, L. Jin, Y. Ran, and B. O. Guan, 'High-birefringence microfiber Sagnac interferometer based humidity sensor', Sensors Actuators, B Chem., 2016, doi: 10.1016/j.snb.2016.03.102.

[15] A. N. Starodumov, L. A. Zenteno, D. Monzon, and E. De La Rosa, 'Fiber Sagnac interferometer temperature sensor', Appl. Phys. Lett., 1997, doi: 10.1063/1.119290.

[16] B. Wu, C. Zhao, B. Xu, and Y. Li, 'Optical fiber hydrogen sensor with single Sagnac interferometer loop based on vernier effect', Sensors Actuators, B Chem., 2018, doi: 10.1016/j.snb.2017.09.124.

[17] S. C. Warren-Smith, R. M. André, J. Dellith, T. Eschrich, M. Becker, and H. Bartelt, 'Sensing with ultra-short Fabry-Perot cavities written into optical micro-fibers', Sensors Actuators, B Chem., 2017, doi: 10.1016/j.snb.2017.01.081.

[18] G. Brambilla, 'Optical fibre nanotaper sensors', Opt. Fiber Technol., 2010, doi: 10.1016/j.yofte.2010.08.009.

[19] J. Lou, Y. Wang, and L. Tong, 'Microfiber optical sensors: A review', Sensors (Switzerland). 2014, doi: 10.3390/s140405823.

[20] M. Ding, P. Wang, and G. Brambilla, 'A microfiber coupler tip thermometer', Opt. Express, 2012, doi: 10.1364/oe.20.005402.

[21] Y. J. Rao, 'In-fibre Bragg grating sensors', Measurement Science and Technology. 1997, doi: 10.1088/0957-0233/8/4/002

[22] Y. Bai, Y. Miao, H. Zhang, and J. Yao, 'Simultaneous Measurement of Temperature and Relative Humidity Based on a Microfiber Sagnac Loop and MoS2', J. Light. Technol., 2020, doi: 10.1109/JLT.2019.2947644.

[23] F. Wei et al., 'Magnetic field sensor based on a tri-microfiber coupler ring in magnetic fluid and a fiber bragg grating', Sensors (Switzerland), 2019, doi: 10.3390/s19235100.

[24] R. Tewari and K. Thyagarajan, "Analysis of tunable singlemode fiber directional couplers using simple and accurate relations', J. Light. Technol., 1986, doi: 10.1109/JLT.1986.1074731.

[25] G. Brambilla, V. Finazzi, and D. J. Richardson, 'Ultra-low-loss optical fiber nanotapers', Opt. Express, 2004, doi: 10.1364/opex.12.002258.

[26] J. A. Corsetti, W. E. Green, J. D. Ellis, G. R. Schmidt, and D. T. Moore, 'Simultaneous interferometric measurement of linear coefficient of thermal expansion and temperature-dependent refractive index coefficient of optical materials', Appl. Opt., 2016, doi: 10.1364/ao.55.008145.

Fangfang Wei received the M.Sc. degree in communication and information system from Tianjin University of Technology, Tianjin, China, in 2012. She is working toward the Ph.D. degree at the Photonics Research Centre, School of Electrical and Electronic Engineering, Technological University Dublin, Dublin, Ireland. Between 2012 and 2015, she was an Optoelectronic Research and Development Engineer with LUSTER Light Vision Tech. Co., Ltd., Beijing, China. Her research interests include optical fiber sensors, microfiber couplers, optical all-fiber lasers, magnetic field sensors, optical waveguides, and fabrication \& packaging of optical devices.

Dejun Liu received the M.Sc. degree in materials science and engineering from Chongqing University, Chongqing, China. He received the Ph.D. degree from Photonics Research Centre, Technological University Dublin, Dublin, Ireland, in 2018. He was an Engineer with Huawei Technology Limited Company, from 2012 to 2013. He is currently working as research fellow at Shenzhen University, Shenzhen, China. His current research interest focuses on tapered optical microfibers for sensing applications.

Zhe Wang received the B.Sc. Degree from Qufu Normal University, Qufu, China, in 2014, and the M.Sc. Degree in optoelectronics from Ningbo University, Ningbo, China, in 2018. He is currently working towards the Ph.D. degree at the Photonics Research Centre, Technological University Dublin, Dublin, Ireland. His research interest includes micro-resonator based biosensor, thermal sensor, and gas sensor.

Zhuochen Wang received the B.S. degree in measurement and control technology and instrument from Southwest Jiaotong University, Chengdu, China. He is currently working toward the Ph.D. degree at the Photonics Research Centre, Technological University Dublin, Ireland. His current interest focuses on WGMs optical micro-resonator for temperature sensing.

Gerald Farrell is the Founder and the former Director of the TU Dublin Photonics Research Centre, Technological University Dublin, Dublin, Ireland. He leads a multinational research team of researchers focusing on several areas of optical fiber sensing research, for example, microfiber and nanofiber sensors for chemical and biosensing and sensors for composite materials. He has led several long-term research collaborations with research groups in China, Poland, and Australia. He was the Director for several years with the startup company PX Instrument Technology, focusing on the design of optical fiber system test and measurement technologies. He was also the Dean of the College of Engineering and Built Environment, Technological University Dublin. He has been involved in a number of research networks, such as the EU COST Action on Optical Sensors and the European Photonics Industry Consortium. $\mathrm{He}$ is a Visiting Professor with the Beijing University of Posts and Telecommunications, Beijing, China, and with the Harbin Engineering University, Harbin, China. He has more than 330 publications in the area of photonics and is an Associate Editor and a Referee for a range of Photonics journals and has been a Member of a number of international photonics conference technical committees.

Qiang Wu received the B.S. and Ph.D. degrees from Beijing Normal University and Beijing University of Posts and Telecommunications, Beijing, China, in 1996 and 2004, respectively. From 2004 to 2006, he worked as a Senior Research Associate in City University of Hong Kong. From 2006 to 2008, he took up a research associate post in Heriot-Watt University, Edinburgh, U.K. From 2008 to 2014, he worked as a Stokes Lecturer at Photonics Research Centre, Dublin Institute of Technology, Ireland. He is with Faculty of Engineering and Environment, Northumbria University, Newcastle Upon Tyne, United Kingdom; Key Laboratory of Nondestructive Test (Ministry of Education), Nanchang Hangkong University, Nanchang 330063, China. His research interests include optical fiber interferometers for novel fiber optical couplers and sensors, nanofiber, microsphere sensors for bio-chemical sensing, the design and fabrication of fiber Bragg grating devices and their applications for sensing, nonlinear fibre optics, surface plasmon resonant and surface acoustic wave sensors. He has over 200 journal publications in the area of photonics and holds 3 invention patents. He is an Editorial Board Member of Scientific Reports, an Associate Editor for IEEE Sensors Journal and an Academic Editor for Journal of Sensors.

Gang-ding Peng received the B.Sc. degree in physics from Fudan University, China, in 1982, the M.Sc. degree in applied physics, and the Ph.D. degree in electronic engineering from Shanghai Jiao Tong University, Shanghai, China, in 1984 and 1987, respectively. From 1987 to 1988, he was on the faculty of the Jiao Tong University and from 1988 to 1991, he was with the Optical Sciences Centre of the Australian National University, Canberra. From 1992 to 1996, he was a Queen Elizabeth II Fellow and is presently a Senior Lecturer with the School of Electrical Engineering \& Telecommunications, University of New South Wales, Sydney, Australia. His research interests include optical fiber devices, polymer optical fibers, and nonlinear optics.

Yuliya Semenova received the M.E. degree from Lviv Polytechnic National University, Lviv, Ukraine, in 1992, and the Ph.D. degree in physics of liquid crystals from the Ukrainian Academy of Sciences, Kiev, Ukraine, in 1999. Between 1997 and 2001, she worked as a Researcher with the Faculty of Electro-Physics, Lviv Polytechnic National University. Since 2001, she has been with the School of Electrical and Electronic Engineering, Technological University Dublin, Dublin, Ireland. Currently, she is a Professor with the School and the director with the Photonics Research Centre. She has more than 270 publications in academic journals in the area of photonics and holds one invention patent. Her current research interests include liquid crystals, photonics, and fiber optic sensing. She is an Associate Editor for Electronic Letters and a Guest Editor for the IEEE Sensors Journal. 